\title{
Photon trapping and energy transfer in multiple-dye plastic matrices: an efficient solar-energy concentrator
}

\author{
B. A. Swartz, T. Cole, and A. H. Zewail \\ Arthur Amos Noyes Laboratory of Chemical Physics, California Institute of Technology, Pasadena, California 91125 \\ Received May 6, 1977
}

\begin{abstract}
Experiments are described illustrating enhanced photon trapping and efficient energy transfer in mixed-dye planar solar concentrators containing, for example, Rhodamine $6 \mathrm{G}$ and Coumarin 6 . These concentrators intercept more of the solar spectrum to give an enhanced photon-flux gain that exceeds the single-dye concentrator. It is also shown that the energy absorbed by the donor dye is transferred efficiently into the emitting acceptor by two competing processes.
\end{abstract}

Of the proposed solar-energy technologies, photovoltaic conversion offers the unique advantages of direct conversion from photons to electrical energy combined with simplicity, low maintenance, and high redundancy. Important problems related to the improvement of photovoltaic conversion efficiency are (a) how to maximize the fraction of the solar spectrum collected and (b) how to improve the methods for coupling solar photons to the photovoltaic junction. A new type of solar collector has recently been proposed, $, 1,2$ based on light-pipe trapping of molecular fluorescence, that can potentially aid in the solution of both (a) and (b). In this Letter we report on a planar solar concentrator (PSC) of this type having substantially improved efficiency through the use of multiple fluorescent dyes. This multiple-dye planar solar concentrator $\left(\mathrm{PSC}_{m}\right)$ is more efficient than a single-dye planar solar concentrator $\left(\mathrm{PSC}_{s}\right)$ because it can intercept more of the solar spectrum using donor dyes and transfer the energy efficiently to an acceptor dye whose energy of emission can be matched to the maximum efficiency wavelength of the solar cell.

The operation of a PSC may be summarized as follows: A flat plate of a transparent material (host) is impregnated with guest molecules possessing a highly efficient luminescence. Solar photons incident on the top of the plate, whose wavelengths lie in the absorption band of the guest, are absorbed and subsequently reemitted at longer wavelengths. The direction of reemission being random, Snell's law dictates that a large fraction (e.g., 0.74) of the re-emitted photons are trapped by total reflection within the plate. Successive reflections carry the luminescent photons to the edge of the plate, where they are coupled to a strip of photovoltaic converters. If three fourths of the entire solar radiant flux of wavelength less than the bandgap wavelength of the semiconductor could be absorbed and re-emitted within a PSC, the edge photon flux would be greater than the flux entering the top of the device by three fourths of the ratio of the top area to edge area. Important advantages of the PSC are: It does not have to be steered to track the sun; it is nearly as efficient in diffuse as in direct sunlight; it reduces heat dissipation in the edge-mounted solar cells, since the excess energy of short-wavelength photons is dissipated over the entire area of the PSC.

A simple embodiment of this concept is a sheet of a transparent plastic of good optical quality [e.g., polymethyl methacrylate (PMMA)], in which an efficient laser dye is dissolved. Previous work ${ }^{1,3}$ has shown that the photon flux gain $G_{P}$ at the edge of a PSC is given by

$$
G_{P}=\left(A_{F / A_{C}}\right) Q_{A} \eta Q_{C} .
$$

$A_{F}$ and $A_{C}$ are the areas of the face and edges of the PSC, respectively. $Q_{C}$, as defined by Lambe and Weber, ${ }^{1}$ is the efficiency of collection of luminescent photons at the edge of the PSC, $\eta$ is the quantum efficiency of the luminescent process, and $Q_{A}$ is the efficiency of absorption of solar photons:

$$
Q_{A}=\frac{\int_{0}^{\lambda_{c}} N(\lambda)[1-\exp \alpha(\lambda) d] d \lambda}{\int_{0}^{\lambda_{c}} N(\lambda) d \lambda},
$$

where $N(\lambda)$ is the photon flux in the solar spectrum, $\lambda_{c}$ is the cutoff wavelength of the photcvoltaic device, $d$ is the pathlength through the PSC, and $\alpha(\lambda)$ is the dyeabsorption coefficient. For an optimum ${ }^{3} \mathrm{PSC}_{s}(\mathrm{Rh} 6 \mathrm{G})$, $Q_{A} \sim 15 \%$. Since the absorption levels of Rh6G and other efficient laser dyes in $\mathrm{PSC}_{s}$ cover only a small fraction of the visible spectrum, the value of $A_{F} / A_{C}$ must be made extremely large, i.e., $\approx 10^{3}$, to achieve good overall photon-flux gain.

The $\mathrm{PSC}_{m}$ overcomes this problem by coupling or cascading the fluorescence of one dye to the absorption of another. In fact, by choosing the right set of dyes, it is feasible to absorb nearly all of the visible spectrum of the sun. Provided efficient energy transfer occurs, the device will emit in a narrow band near the wavelength of maximum efficiency of the photovoltaic cell.

Planar solar concentrators of polymethyl methacrylate doped with laser dyes were cast from solutions of the monomer. The concentrators, which have good optical quality, were sanded to size $(7 \mathrm{~cm} \times 6.5 \mathrm{~cm} \times$ 


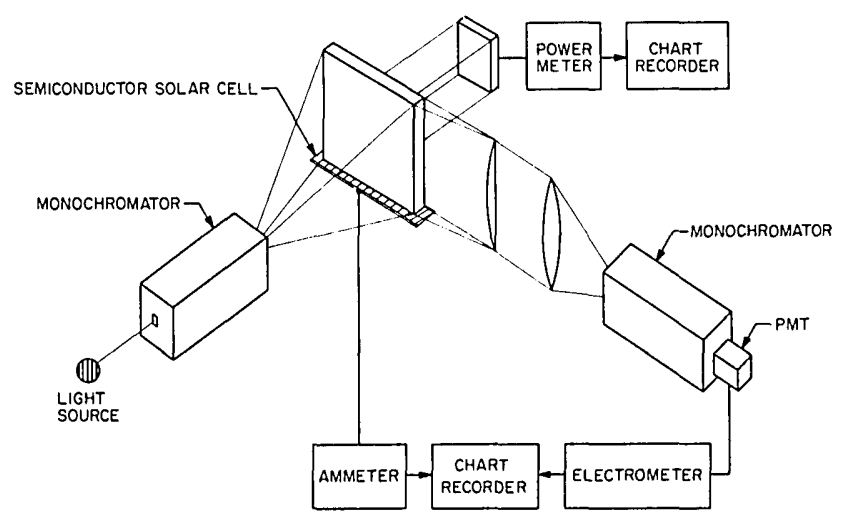

Fig. 1. Experimental setup for the measurements of optical excitation, emission spectra, and the photovoltaic current. In all these experiments care was taken to mask the detectors from scattered light.

$0.18 \mathrm{~cm})$ and the edges polished with cerium oxide. Coumarin 6 (C6) and Rhodamine (Rh6G) dyes were used at concentrations of $\mathrm{Ca} 10^{-4} \mathrm{M}$.

Optical excitation and emission spectra were taken of the concentrators, using the apparatus diagrammed in Fig. 1. An Oriel 150-W Xe lamp and 0.25-m Jarrell-Ash monochromator were used for the excitation. The edge emission was detected through a $0.5-\mathrm{m}$ (or a 1.8-m) Jarrell-Ash monochromator by a photomultiplier tube. Current excitation spectra were taken by monitoring with a Keithley picoammeter the current produced from a Si solar cell (Spectra Lab.) coupled to one edge of the concentrator. A thin film of index-matching material was placed between the plastic $(n=1.51)$ and the photocell, eliminating a highly reflective plastic-air interface. Measurements of the total current produced with broadband excitation were accomplished by simply replacing the exciting monochromater with a diffuser. The power incident on and transmitted through the concentrators was measured with a UDT $(40 \times)$ power meter.

Figure 2 depicts the emission and the optical excitation spectra of Rh6G and C6 in the PMMA. The spectra mimic the known solution spectra ${ }^{4}$ of these dyes, except for a shift in the emission band to longer wavelength.

Figure 3 gives the emission spectrum of $\mathrm{PSC}_{m}$ (Rh6G/C6), using monochromatic excitation at $\lambda=4700$ $\AA$, which is the absorption maximum for $\mathrm{C} 6$. This indicates an efficient energy transfer, since there is essentially no detected emission characteristic of $\mathrm{C} 6$ with the edge-mounted detector. As we shall discuss, part of the energy transfer mechanism in the plastic is similar to that observed in dye solutions that enhance the gain spectrum of dye lasers. ${ }^{5}$

We have measured the optical excitation spectra of the $\mathrm{PSC}_{m}$ by using two detection schemes, a photomultiplier in connection with the monochromator at $\lambda$ $=5840 \AA$ and a broadband silicon solar cell edgemounted to the concentrator. As shown in Fig. 4, the results are essentially identical and show that the excitation spectrum of the $\mathrm{PSC}_{m}$ is basically the sum of the two absorption spectra shown in Fig. 2 for the individual dyes. This is an important conclusion because it indicates that the relative efficiency of $\mathrm{PSC}_{m}$ to $\mathrm{PSC}_{s}$ is essentially in proportion to the integrated absorption area. This result was confirmed by measuring the ratio of the photocurrent of the silicon solar cell edge-coupled for the two concentrators with identical white illumination. This ratio, which corresponds to $Q_{A}$ (Rh6G/ C6) $/ Q_{A}$ (Rh6G), was found ${ }^{6}$ for Rh6G/C6 versus $\mathrm{Rh} 6 \mathrm{G}$ to be $2.0 \pm 0.3$. Since this value is close to the ratio of excitation curve areas for the $\mathrm{PSC}_{m}$ to $\mathrm{PSC}_{s}$, we conclude that the energy transfer in the $\mathrm{PSC}_{m}$ is quite efficient, and the principle of the $P S C_{\mathrm{m}}$ has been demonstrated.

If a series of dyes covering the visible spectrum is used, a $\mathrm{PSC}_{m}$ can have a $Q_{A}$ approaching 1 . Thus a high concentration ratio can be achieved with a smaller-ratio $A_{F} / A_{C}$ than a $\mathrm{PSC}_{s}$. This improvement could be as much as a factor of 8 over a Rh6G PSC. This relaxes the need for extremely low scattering in the matrix, low self absorption in the emitting dye, and optical perfection of the PSC surfaces. An overall flux gain of 100 should be possible in an optmized $\mathrm{PSC}_{m}$ compared with the calculated ${ }^{3}$ optimum gain of 14 for a $\mathrm{PSC}_{s}(\mathrm{Rh} 6 \mathrm{G})$ with $\mathrm{Q}_{A}=0.13$.

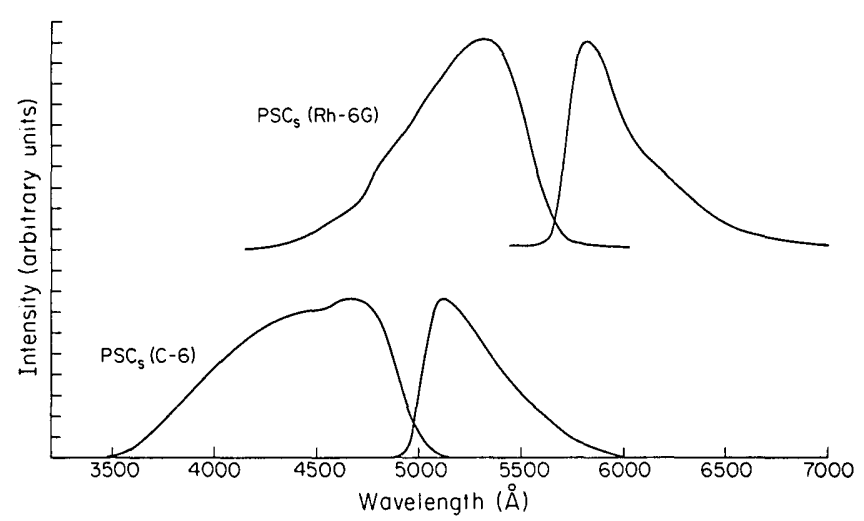

Fig. 2. Optical excitation and emission spectra of Rh6G and C6 in PSC's. Note the broadening of the absorption when compared with the emission spectra. All these spectra are corrected for the exciting light and the detector.

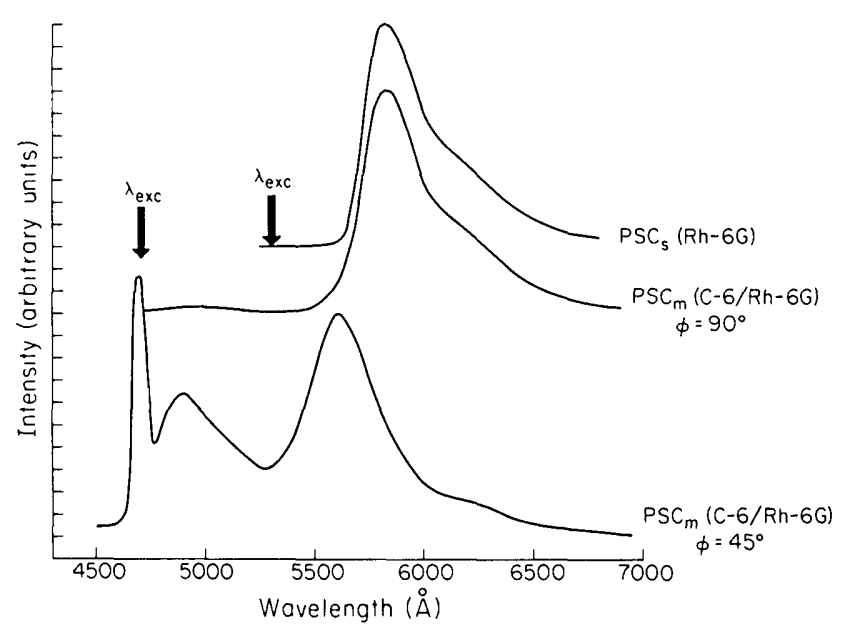

Fig. 3. Top: emission spectrum of Rh6G in the PSC; $\lambda_{\text {excit }}$ $=5300 \AA$. Middle: emission spectrum of $\mathrm{PSC}_{m}(\mathrm{Rh} 6 \mathrm{G} / \mathrm{C} 6)$ at collection angle of $90^{\circ} ; \lambda_{\text {excit }}=4700 \AA$. Bottom: emission spectrum of $\mathrm{PSC}_{m}(\mathrm{Rh} 6 \mathrm{G} / \mathrm{C} 6)$ at collection angle of $\sim 45^{\circ}$; $\lambda_{\text {excit }}=4700 \AA$. The sharp band at $4700 \AA$ is the reflected exciting light. 
Two processes may participate in the energy transfer in the $\mathrm{PSC}_{m}$ system, simple radiative transfer and donor-acceptor transfer via the Förster ${ }^{7}$ dipole-dipole mechanism. The Förster theory gives a critical distance $R_{0}$ and a critical concentration of acceptor $C_{0}(A)$, which are given by

$$
R_{0}^{6}=9 \times 10^{-25}\left\langle x^{2}\right\rangle \Phi_{D} F(\bar{\nu}) n^{-4}
$$

and

$$
C_{0}(A)=8.35 \times 10^{-3} N_{0}^{-1} R_{0}^{-3},
$$

where $N_{0}$ is Avogadro's number and $\Phi_{D}$ is the donor quantum yield. The function $F(\bar{\nu})$ is the overlap between the emission spectrum $f_{D}{ }^{(e)}$ of the donor and the absorption spectrum of the acceptor $f_{A}{ }^{(a)}$ :

$$
F(\bar{\nu})=\int f_{D}{ }^{(e)} f_{A}{ }^{(a)} \bar{\nu}^{-4} \mathrm{~d} \bar{\nu} .
$$

Knowing the index of refraction of the plastic $(n)$ and assuming the geometrical factor $\left\langle x^{2}\right\rangle=2 / 3$, we estimate that the Förster-type transfer will play the major role at concentrations higher than $\sim 10^{-3} M$. A different spatial distribution of donor and acceptor molecules could modify this conclusion, however. The radiative transfer, on the other hand, takes place in dilute systems and depends crucially on the photon pathlength within the sample.

These considerations prompted an experiment, the results of which are shown in Fig. 3 (bottom). In this experiment the $\mathrm{PSC}_{m}$ is excited by a beam of light making an angle $\Phi=45^{\circ}$ with the top surface, and the light emerging from the top surface is collected by a spectrometer that is also at an angle of $45^{\circ}$ to the top surface. In addition to the $\mathrm{Rh} 6 \mathrm{G}$ emission band that appears in the edge-mounted collector spectrum (Fig. 3 , middle), the $\mathrm{C} 6$ emission band also appears. Second, the spectra of both Rhodamine and Coumarin in the $45^{\circ}$ experiment are shifted from their positions in the edge-mounted experiment to shorter wavelengths. The emission-band shift can be accounted for, at least in part, by reduced self absorption in the dyes, ${ }^{3,7}$ but the relatively strong $\mathrm{Rh} 6 \mathrm{G}$ donor emission, even though the
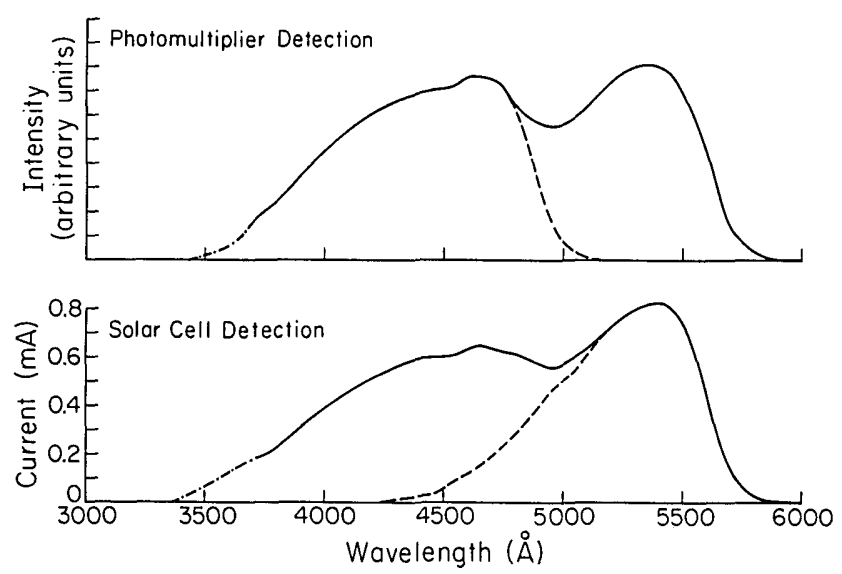

Fig. 4. Optical excitation spectra of $\mathrm{PSC}_{m}$ (Rh6G/C6): top, photomultiplier detection; bottom, silicon-cell detection. The two spectra were taken for edge-mounted geometry, and they were corrected for the exciting light and the detector. Dotted lines indicate excitation profiles for the individual dyes in $\mathrm{PSC}_{s}$ 's. optical pathlength for donor photons in this experimental arrangement is rather short, would indicate some transfer via the Förster mechanism. One would not expect the Förster mechanism to be dominant at the concentrations $\left(\sim 10^{-4} M\right)$ used in these $\mathrm{PSC}_{m}$ 's. Experiments ${ }^{8}$ by Logan et al. ${ }^{9}$ and Jortner et al. ${ }^{10}$ on radiative trapping effects in neat crystals of anthracene, where Förster channels do not exist, show the presence of the radiative transfer. Detailed polarization and time-resolved luminescence experiments are required over a range of dye concentrations to elucidate fully the energy-transfer mechanisms in these systems. Such experiments are currently in progress in this laboratory.

The conclusions to be drawn from the foregoing results are the following: Homogeneous mixed-dye PSC's are practical and can improve the gain of the concentrator by at least the ratio of the sum of the absorption areas of the multiple-dye to single-dye concentrators. Furthermore, the mechanisms of radiative- and Förster-energy transfer appear to be operative in the $\mathrm{PSC}_{m}$ system. It is clear that subsequent work should also focus on extension to $\mathrm{PSC}_{m}$ 's containing three or more dyes. Such multiple-dye systems will require careful study of the intermolecular-energy-transfer mechanisms. Studies of the concentration dependence of energy transfer are in progress.

\section{References}

1. W. H. Weber and J. Lambe, Appl. Opt. 15, 2299, (1976).

2. A scheme similar to the PSC was earlier proposed for collecting light in scintillation counters. See W. A. Shurcliff, J. Opt. Soc. Am. 41, 209 (1951); R. L. Garwin, Rev. Sci. Instrum. 31, 1010 (1960); G. Keil, J. Appl. Phys. 40, 3544 (1969).

3. J. E. Levitt and W. H. Weber, Appl. Opt. (to be published).

4. F. P. Schafer, ed., Dye Lasers (Springer-Verlag, New York, 1973).

5. See, for example, C. E. Moeller, C. M. Verber, and A. H. Adelman, Appl. Phys. Lett. 18, 278 (1971); S. A. Ahmed, J. S. Gergely, and D. Infante, J. Chem. Phys. 61, 1584 (1974); T. Unisu and K. Kajiyanea, J. Appl. Phys. 47, 3573 (1976).

6. The measurement of the current is consistent with the reported ratio when the $\mathrm{PSC}_{m}$ and $\mathrm{PSC}_{s}$ were exposed to sunlight. These preliminary results were corrected for scattered light by using a plastic that does not have any dye but possesses the same dimensions as the PSC under investigation.

7. Th. Förster, Discus. Faraday Soc. 27, 7 (1959).

8. J. Jortner has brought our attention to the work done on anthracene crystals. ${ }^{9,10}$ Thick crystals give a lifetime of $16 \mathrm{nsec}$ at room temperature and $8 \mathrm{nsec}$ at $70 \mathrm{~K}$. Because thin anthracene crystals will not absorb one photon uniformally, two-photon excitation ${ }^{10}$ of crystals of variable thickness gave different lifetimes, $27 \mathrm{nsec}$ for $1-\mathrm{cm}$-thick and $12 \mathrm{nsec}$ for $1-\mu \mathrm{m}$-thick crystals. These results were interpreted in terms of radiation trapping by the (thermally populated) hot vibrational bands.

9. L. M. Logan, I. H. Munro, D. F. Williams, and F. R. Lipsett in Molecular Luminescence, E. Lim, ed. (Benjamin, New York, 1969), p. 773.

10. J. Burak and J. Jortner (unpublished results). 